\title{
An Effective Integrated Approach for the Teaching of Power Electronics to Part-time Engineering Students
}

\author{
Yue-Chung Wong \\ Hong Kong Technical College (Tsing Yi)
}

\begin{abstract}
The task of teaching power electronics to students in a part-time electrical engineering course is difficult and challenging. This paper aims to share the experience gained in the development of an effective approach for teaching the subject. In the beginning of the paper, the difficulties involved are introduced. Then, the problems encountered in teaching the subject in the first two years are described and the reasons for which the solutions developed in that period were ineffective are analyzed. Finally, the events that led to the development, and the details of the effective integrated approach are presented.

In this integrated approach, various student learning and studying problems were tackled. First of all, a purpose-built workbook has been developed. This workbook is mainly a simplified version of the student textbook. But, summary of lengthy sessions and comparisons of related power electronic circuits are added throughout the set of notes to help students to focus upon the important areas of Power Electronics. Second, lecture and tutorial time are combined to provide opportunity for the students to work on simple and short questions specially designed to help them to clarify the topics just taught in a lecture class. This is to ensure that the whole class is progressing at the same pace. Third, teaching staff always actively help the students to solve their problems during tutorial. Fourth, computer simulation experiments have been developed to complement lecture materials. The details of the integrated approach will be described fully in the main paper so that adaptation/modification of the ideas developed can be done easily for similar situations.
\end{abstract}




\section{Introduction}

Power Electronics is a subject taught in the second year of a 3-year part-time evening Higher Certificate Electrical Engineering course offered by the Electrical \& Communications Engineering Department of the Hong Kong Technical College (Tsing Yi). It has been taught for 3 years now. Teaching this subject in the evening course has been a difficult and challenging task for a number of reasons. Firstly, the multi-disciplinary nature of the subject requires the students to be familiar with the basic concepts and background of a number of other subjects including electromagnetic theory, network theory, feedback control theory, and both analog and digital electronics. For instance, power electronics involves the generation of electromagnetic interference as well as the working principles of the various types of power conversion and electrical motor drive circuits. Secondly, as most of the students taking the course are practicing engineers/technicians in the various sectors of the Hong Kong electrical industry, their electronics engineering knowledge/contacts/background is varied and, perhaps, weak. Thirdly, as part-time students, they do not have much free time to study consistently throughout the year, considered that they have to attend 3 evenings of scheduled classes (from 6:30 p.m. to 9:30 p.m.) per week, plus their employment and other necessary commitments. Although, this third reason is not uncommon among part-time students, nevertheless when coupled with an inappropriate engineering background, the subject does become very hard for the students to grasp and for the lecturer to teach. Before the integrated approach was developed, a great deal of the time of a lecture had to be used to recap or even re-teach what had been taught in previous lectures. What follows is a discussion of the evolution of teaching power electronics in an integrated approach with much higher success rate.

\section{Problems and solutions in the first 2 years of teaching the subject}

During the year (1994-95) when the subject was taught for the first time, it was found out that, almost right from the beginning, the subject was difficult for most of the students judging from their performance in laboratory, tutorial and lecture classes. In order to help the students to learn the subject, explanations of the working principles of the power electronics circuits were presented with extra care and usually in a step by step manner in lecture classes. Additional time was made available for revisions of the materials taught to assist the students in passing their examination of the subject. Nevertheless, 49 out of 178 students failed in their first attempt to pass the examination, a very high failure rate of $27.5 \%$. Unfortunately, at that time the high failure rate could only be identified as relating to the multi-disciplinary nature of the subject and the students' inappropriate engineering background. The crucial factor that the students were lacking time to properly study the subject was missed by the author. As a result, the remedial measures introduced in the following year was not as effective as expected.

Before the beginning of the second year that the subject was taught, a full set of special tutorial problems, in addition to the usual numerical tutorial problems, was prepared. They were designed to guide the students to study the subject by asking the students to answer a series of short questions aimed to help clarify concepts that were difficult for them. Although the good intention of these studying guides was understood and appreciated by most of the students, very few of them showed sufficient self-initiatives to work out these tutorial problems at home. It seemed that they would not study unless given specific assignments and would not revise unless 
there was a formal test. This kind of studying effort was a big disappointment to the teaching staff involved. Consider that these students were mature individuals, so it would be quite natural to expect them to know how to learn the material.

A new strategy was needed to tackle the problems in teaching power electronics to part-time electrical engineering students. With the helpful suggestions/ideas from the end of the year student feedback and review discussions among the teaching staff involved, a new integrated approach was formulated. This work was performed during the summer vacation in preparation for the third year that the subject would be taught.

The outcome of the new approach was very encouraging: the students learned the material much better and the examination failure rate went down to $13.5 \%$, a big improvement over the previous two years.

\section{The new integrated approach}

In order to deal with the various problems mentioned, several methods were integrated. First, a purpose-built power electronics workbook was developed to help the students' learning and studying. Second, lecture and tutorial time were combined so that timely short questions can be given to the students to help clarify concepts just taught during a lecture class. Third, more emphasis was placed upon Continuous Assessment.

\subsection{Purpose-built workbook}

In this integrated approach, a purpose-built power electronics workbook has been developed to complement the subject textbooks recommended to the students. In this workbook, all complicated concepts, and circuit operations of the original text have been made more understandable by replacing the lengthy paragraphs of descriptions in the text with step-by-step simple English explanations. This has addressed both the problem of insufficient background and the English language problem. In addition, this workbook also includes, worked examples, tutorial questions, comparisons between various related or similar power electronic circuits, and summary of lengthy sections of text. For example, at the end of the section about 3-phase 3pulse rectifiers, the main points were summarized in the following manner:

\section{3 phase, 3 pulse rectifier circuit operation (Page 14 to page 17)}

\section{Main Points:}

The voltage of $\mathbf{K}$ always follows the highest line voltage of the three phases.

$\mathrm{L}$ is assumed very large, so that output current can be considered having no ripple at all.

The diodes used are ideal diodes with zero switching delay.

Hence, the shape of the output current is rectangular.

But, this causes electromagnetic interference.

UF of the transformer is only $74 \%$, as each phase conducts $1 / 3$ of the time in every cycle.

Because it points out clearly the main points of important areas of the subject, this workbook was well received by the students. 


\subsection{Combination of lecture and tutorial time}

The problems associated with the students not having enough free time were addressed mostly by the combination of lecture and tutorial time. After each major item of discussion, students would be then given time in the class and asked to answer some simple and short questions of minor difficulty, but directly relevant to the topic just discussed. These short questions have been carefully designed for guiding students to think, and follow through in discussions, and clarify explanations made during lectures. For example, at the end of the notes about a sub-topic: Diode and Charger, the following questions are added in the workbook:

\section{Diode and Charger}

1. With reference to Page 1, explain why diode can be used as electronic switch in power electronic circuit.

2. With reference to Page 3, explain why does I flow only between $t_{1}$ and $t_{2}$ in a cycle and not in any other times.

3. With reference to Page 3, explain the error in the waveform.

4. With reference to Page 3, state the purpose of $\mathrm{R}$ in the Charger circuit.

5. With reference to Page $5 \&$ 6, it can be seen that inductor can store and release energy. Explain in what way this property is useful.

6. With reference to Page 5 \& 6, explain why does the current become zero at $t_{3}$.

7. With reference to Page 5 \& 6, explain the purpose of finding $A(+)$.

The teaching staff was pro-active in helping students to solve problems when required, as this was a way to encourage the students to work harder. At the end of a specified time, the students have to submit their answers. Answers of these tutorial questions were then explained to the whole class followed by the distribution of printed copies of the answers. The purpose of this work is to ensure that the whole class can understand the lectures and progress at more or less the same pace.

\subsection{Continuous Assessment and Short Tests}

In the first lecture of the year, the students were informed some of the class-work would contribute towards their Continuous Assessment (CA) marks. The CA has 3 components: inclass or take-home assignments, tests, and lab work. The weighting of the CA mark was and still is $30 \%$ of the total marks of the subject. And the weighting of examination was and still is $70 \%$. In order to ensure the students would work all the assignments, they were not informed as to which ones would be applied to their grades. Short tests were given at a higher frequency than the previous years.

These new approaches were adopted because they were considered necessary. They were welcomed by most of the students. The main reason for their support was that the students had more confidence in their ability to pass the examination of the subject. As the students became more confident in themselves, they were more willing to work harder, a kind of positive feedback effect. This was a big reward for the teaching staff involved. The teaching staff's experience proved that the right solution to any problem can only be found and developed after the real reasons of the problems are understood. 


\section{Current Developments}

Encouraged by the success of these new methods, a full set of new experiments were developed. The new experiments [1-4] designed are based upon MicroSim Design Center (PSpice) Evaluation Version 6.0. There are several advantages in using the MicroSim Design Center for teaching in a laboratory class, and in designing the experiments based upon its Evaluation Version. Firstly, the package itself is very powerful. With the package, it is easy to extract useful information about the operations of the various power electronic circuits taught in lecture class. The visualization of the waveforms helped the students to understand better than the text descriptions of the inter-related actions of the various circuit components. Secondly, with the high computation speed of today's personal computers (PC), it is practical to encourage students to modify circuit parameters on an ad hoc basis during lab-class. This "what if" approach is very educational. Lastly, the Evaluation Version of PSpice is a freeware. The students have the opportunity to further study the circuits in the experiments or other circuits taught in lecture at home at their own pace. Hopefully, the students' learning of power electronics would be improved further this year by these complementary computer simulation exercises.

\section{Conclusion}

This paper was written with the aim to share the experience gained in the development of an effective approach for teaching the subject of power electronics. In the beginning of the paper, the difficulties involved were introduced. Then, the problems encountered in teaching the subject in the first two years were described and the reasons for which the solutions developed in that period were ineffective were analyzed. Finally, the events that led to the development, and the details of the effective integrated approach were presented.

\section{References}

1. Thomas H. Sloane, "Laboratories for an Undergraduate Course in Power Electronics". IEEE Transactions on Education, Vol. 38, No. 4, November 1995.

2. Simon S. Ang, "A Practice-Oriented Course in Switching Converters". IEEE Transactions on Education, Vol. 39, No. 1, February 1996.

3. David A. Torrey, "A Project-Oriented Power Electronics Laboratory". IEEE Transactions on Power Electronics, Vol. 9, No. 3, May 1994.

4. Daniel W. Hart, "Circuit Simulation as an Aid in Teaching the Principles of Power Electronics". IEEE Transactions on Education, Vol. 36, No. 1, February 1993. 